\title{
Measuring the Vibrational Density of States of Nanocrystal-Based Thin Films with Inelastic X-ray Scattering
}

\section{Journal Article}

Author(s):

Yazdani, Nuri (D); Nguyen-Thanh, Tra; Yarema, Maksym (1); Lin, Weyde (1); Gao, Ramon; Yarema, Olesya (D); Bosak, Alexey; Wood, Vanessa (1)

Publication date:

2018-04-05

Permanent link:

https://doi.org/10.3929/ethz-b-000258150

Rights / license:

In Copyright - Non-Commercial Use Permitted

Originally published in:

The Journal of Physical Chemistry Letters 9(7), https://doi.org/10.1021/acs.jpclett.8b00409 


\section{Measuring the Vibrational Density of States of}

\section{Nanocrystal-Based Thin Films with Inelastic X-}

\section{Ray Scattering}

Nuri Yazdani,1 Tra Nguyen-Thanh,2 Maksym Yarema, , Weyde M M Lin,1 Ramon Gao, 1

Olesya Yarema, 1 Alexey Bosak,2 Vanessa Wood1

1. Department of Information Technology and Electrical Engineering, ETH Zurich, Zurich, 8092 Switzerland

2. European Synchrotron Radiation Facility, 71, Avenue des Martyrs, F-38000 Grenoble, France 


\section{Abstract}

Knowledge of the vibrational structure of a semiconductor is essential for explaining its optical and electronic properties and enabling optimized materials selection for optoelectronic devices. However, measurement of the vibrational density of states of nanomaterials is challenging. Here, using the example of colloidal nanocrystals (quantum dots) we show that the vibrational density of states of nanomaterials can be accurately and efficiently measured with inelastic xray scattering (IXS). Using IXS, we report the first experimental measurements of the vibrational density of states for lead sulfide nanocrystals with different halide-ion terminations and for $\mathrm{CsPbBr}$ perovskite nanocrystals. IXS findings are supported with ab-initio molecular dynamics simulations, which provide insight into the origin of the measured vibrational structure and the effect of nanocrystal surface. Our findings highlight the advantages of IXS compared to other methods for measuring the vibrational density of states of nanocrystals such as inelastic neutron scattering and Raman scattering.

\section{Table of Contents Figure:}

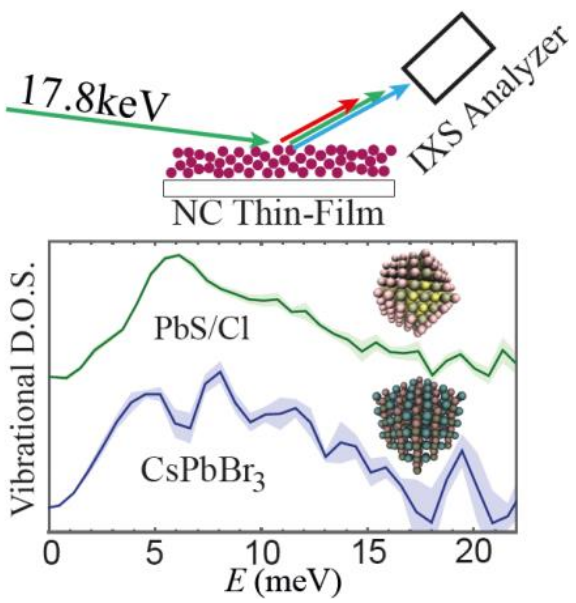

Keywords: vibrational structure, phonon density of states, perovskite nanocrystals, chalcogenide nanocrystals, surface termination, inelastic x-ray scattering 
To fully explain the optical and electronic properties of a semiconductor, it is critical to understand its nuclear dynamics, such as the vibrational density of states $g(E)$, particularly for nanomaterials, where charge carrier energy gain/loss,1-7 and transport8-12, are driven by coupling of the nuclear and electronic degrees of freedom. In colloidally synthesized nanocrystals (NCs), coupling to vibrations can explain experimentally observed large thermal broadening,13,14 fast carrier cooling, 4 and efficient non-radiative recombination across large energy gaps.3

In this work, we show that synchrotron-based inelastic x-ray scattering (IXS) is a powerful way to determine the vibrational structure of nanomaterials, using NCs as an exemplary material. We begin with an overview of methods for measuring vibrational structure and then validate the IXS approach and highlight its specific advantages by assessing PbS NCs terminated with ethanedithiol ligands, which have previously been extensively studied experimentally using $\mathrm{INS}_{3}$ and Raman techniques as well as ab-initio molecular dynamics (AIMD) calculations.4 We then use IXS to perform the first experimental measurements of the vibrational density of states of $\mathrm{PbS}$ NCs with different halide terminations and of $\mathrm{Cs} \mathrm{PbBr} 3$ perovskite $\mathrm{NCs}$.

In general, vibrational structure of a material can be investigated experimentally by using a charge neutral probe (i.e., photons or neutrons) to modify the vibrational state of the sample through scattering or absorption. Absorption based techniques such as infrared spectroscopy and time resolved pump-probe techniques (e.g. time resolved pump-probe x-ray diffraction,15,16 three-pulse photon echo peak shift17) can be used to investigate specific vibrations (1) through direct excitation of the vibration through absorption, or (2) indirect excitation of vibrations through their coupling to an optically driven electronic transition.16 Inelastic scattering of photons or neutrons (Figure 1a) has the advantage over absorption-based optical approaches in that the scattering intensity is independent of the dipole moment of the vibration and depends in a relatively straightforward manner on the scattering cross-section of 
the photons or neutrons with the atoms involved in the vibrations, facilitating the characterization of the entire vibrational spectrum.18-20

Both inelastic neutron scattering (INS) 21 and Raman spectroscopy22-28 have been used in the characterization of the vibrational structure of nanomaterials, however both have intrinsic limitations.

Inelastic neutron scattering (INS) is often considered the gold standard for probing nuclear dynamics of crystals because neutrons have kinetic energies and momentum comparable to those of typical lattice vibrations 18 and has been previously used to study NCs solids,3 INS for NC-based thin films is challenging. First, due to the relatively low flux of neutrons from state of the art neutron sources, large sample sizes are needed to obtain reasonable scattering statistics with INS. For example, $\sim 10 \mathrm{~g}$ of $\mathrm{PbS}$ NCs were needed per sample to achieve reasonable statistics within a $10 \mathrm{~h}$ measurement window, 3 which necessitates scale up of both the synthesis 29 and ligand exchange procedure. Second, extraction of the phonon density of state with INS is challenging if hydrogen $(\mathrm{H})$ atoms are present in the sample. $\mathrm{H}$ has an extremely large neutron scattering cross-section $(\sim 15$ times that of $\mathrm{Pb}), 30$ such that the signal from $\mathrm{H}$ can dominate the inelastic scattering spectrum. This can be particularly problematic for colloidal nanamaterials, where long hydrocarbon-chain ligands are typically employed in the synthesis to maintain colloidal stability.31,32 This can be circumvented by deuteration of the hydrocarbons used in the NC synthesis and ligand exchange; however, this involves additional steps and can be costly given the large sample sizes required for INS. Because the NC surface is highly sensitive to the chemical processing performed on the NCs (either the removal of excess NC ligands following their growth 33,34 or in the processing into densely packed thin films for inclusion in opto-electronic devices35), the vibrational structure of the NCs should ideally be measured on samples treated identically to those used in the application of interest, and not on a sample highly adapted for INS. 
Raman scattering can be applied to thin films. However, in nanomaterials, where the vibrations cannot be assumed to be strictly localized between two neighboring nuclei, the vibrational density of states extracted from inelastic scattering, $g(E, q)$, will depend on the momentum transfer, $q \equiv \vec{p}_{\gamma i}-\vec{p}_{\gamma f}$. In a Raman experiment, the wavelength of the incident photon is much larger than the unit-cell length of the constituent material (e.g., $a P b S=5.936 \AA$ ). The maximum measureable momentum transfer is thus much smaller than reciprocal lattice vectors of the materials, and integration over all possible momentum transfers will sample $g(E, q)$ only about $q=0$ in reciprocal space 36,37

Synchrotron IXS alleviates (1) the sample preparation problems of INS and (2) the $q=0$ limitation of Raman Scattering. First, high brilliance and flux of focused light sources enables the investigation of much smaller sample sizes, and, in the grazing incidence geometry, thin films on the order of tens of nanometers in thickness are sufficient (Figure 1b). In fact, all thin films studied here are made identically to those in actual NC-based devices (in the case of PbS NCs),38,39 or spin-cast on substrates directly from their synthesized solution (in the case $\mathrm{Cs} \mathrm{PbBr} 3$ perovskite $\mathrm{NCs}$ ). Furthermore, the presence of hydrocarbons is not a problem in IXS, where the atomic form factor for inelastic scattering from $\mathrm{H}$ is very low, and increases proportionally to the atomic number.40 Second, synchrotron light sources provide energies in the $10 \mathrm{~s}$ of $\mathrm{keV}$, whose wavelengths are significantly smaller than that of typical lattice vectors. Measurement of the inelastic scattering can thus be carried out for large momentum transfers, greatly enhancing the sampling of $g(E, q)$ over the entire Brillouin zone, as depicted in Figure 1c. For a polycrystalline materials, in the limit of very large $q$, the extracted $g(E)$ integrated over an infinitesimal $q$-range will approach the true $g(E)$, i.e. uniform sampling of $g(E, q)$ over the entire Brillouin zone.20 
We first validate the IXS approach for NCs and confirm its advantages by studying PbS NCs terminated with ethanedithiol ligands. As described in the Methods, thin films of $r=1.6 \mathrm{~nm}$ PbS NCs approximately 40nm-thick are prepared on single crystal [110] diamond substrates (see Supporting Information SI-1 for the absorption spectrum and measured diffraction pattern of the PbS NCs and the ligand exchanged thin films). Diamond is used since its vibrations occur at high energies, minimizing overlap of the vibrational density of states with the sample. The NC thin-film preparation approach is identical to that used in the fabrication of PbS NC-based solar cells.38,39

IXS measurements are performed at the ID28 beamline at European Synchrotron Radiation Facility (ESRF). A schematic of the setup is depicted in Figure 1d. The (999) backscattering reflections of $\mathrm{Si}$ is employed to achieve a monochromatic beam at a wavelength of $0.6968 \AA$ $(17.8 \mathrm{keV})$. This beam impinges on the sample, scatters, and is analyzed by a second $\mathrm{Si}$ monochromator. By varying the relative temperatures of the two Si monochromators to tune their lattice constants and thereby the resulting diffraction angles, one can scan over photonto-sample energy transfers. Here, measurements are performed in $\sim 0.7 \mathrm{meV}$ steps (corresponding to $\sim 16 \mathrm{mK}$ temperature steps for the $\mathrm{Si}$ analyzer), over the range of energies from $E_{\min }=-16 \mathrm{meV}(\sim 22.5 \mathrm{~K})$ to $E_{\max }=35 \mathrm{meV}(\sim 21.3 \mathrm{~K})$, where the negative energy transfer indicates energy loss from the sample. This results in a measured scattering spectra $I_{M}(E)=I_{I N}(E)+I_{E L}(E)$, a sum of both inelastic and elastic scattering, from which we can compute the atomic form factor weighted $g(E)$, i.e. $\tilde{g}(E) \cdot \tilde{g}(E)$ can be related to the partial density of states for each element in the material, $g_{x}(E)$,

$$
\tilde{g}(E) \propto \sum_{X} \frac{\left(f_{X}(q)\right)^{2}}{M_{X}} g_{X}(E),
$$


where $X$ runs over all of the elements in the material, and $f_{X}(q)$ is the atomic form factor of element $X$, which will depend on the momentum transfer $q$ and is a measure of the scattering probability of the x-rays from element $X .40$ The procedure to compute $\tilde{g}(E)$ from the measured scattering $I_{M}(E)$ is based on the method outlined by Bosak and Krisch,20 and Kohn and Chumakov41 and is described briefly in the methods and in detail in Supporting Information, SI-2.

The $\tilde{g}(\mathrm{E})$ calculated from IXS measurements for the PbS/EDT thin film is shown along with the INS data taken on the PbS powder in Figure 2a. Before comparing the IXS and INS data, we consider whether our IXS measurements are as expected. The $g(E)$ calculated using AIMD for a $r=1.2 \mathrm{~nm} \mathrm{PbS} /$ thiol $\mathrm{NC}$ is shown in Figure 2b. From the partial $g_{x}(E)$, we can calculate the atomic form factor weighted $g(E), \tilde{g}(E)$, using the atomic form factors, $f_{X}(q)$, at the measured momentum transfer for the different elements in the PbS/EDT NC-solid ( $f_{P b}(q)=52.8, f_{S}(q)=7.6, f_{C}(q)=1.9, f_{H}(q) \equiv 1$.) following Cromer and Mann.40 Since the relative scattering from $\mathrm{Pb}$ is much higher than the other elements in the sample, $\tilde{g}(E)$ will take the form of the partial density of states of $\mathrm{Pb}, g_{P b}(E)$, which is consistent with the experimental finding in Figure 2a.

Comparing the $\tilde{g}(E)$ measured from IXS and the $g(E)$ measured by INS, 3 we note relatively good agreement. We fit a sum of two Gaussians to low energy ( $0 \mathrm{meV}<\hbar \omega<8 \mathrm{meV})$ portion of the vibrational spectra (dashed lines in Figure 2a) and extract a transverse acoustic (TA) peaks from IXS at $6.32 \pm 0.35 \mathrm{meV}$ and from INS at $6.00 \pm 0.22 \mathrm{meV}$, which are within error of one another and agreement with the value expected from DFT calculations for bulk PbS $(6.05$ $\mathrm{meV})$ in Figure 2c. The Guassian peaked at lower energy $(\sim 3.4 \mathrm{meV})$ in both the IXS and INS data indicate the presence of a set of low frequency modes, which can be attributed to vibrations 
of low-coordinated $\mathrm{Pb}$ atoms on the facets and edges of the NCs.3,4 However, there are fewer low frequency modes in the IXS results compared to INS. We attribute this to the differences in the sample preparation between the two techniques. While the thin-film samples for IXS are prepared using a standardized procedure employed for device fabrication, due to the large quantity of material needed for INS, the INS samples were prepared using an aggressive, solution based ligand exchange procedure, 3 which likely leaves a more disordered surface. We rule out that the difference in the number of low frequency modes is due to different morphologies of NC packing in the thin film sample for IXS and the powder sample for INS. The density of inter-NC vibrations $(3 \rho \mathrm{NCs}-6$, where $\rho$ NCs is the number of NCs per unit volume) will be orders of magnitude smaller than the density of vibrations on the individual NCs ( $\rho \mathrm{NCs}_{2}\left(3 N_{A}-6\right)$, where $N_{A}$ is the number of atoms per NC). The measured $g(E)$ are therefore representative of the $g(E)$ for the individual NCs. As low frequency vibrations can efficiently drive multi-phonon mediated electronic transitions in nanomaterials at temperatures $T \gg \gg \hbar, 4,8,94$ such as non-radiative recombination and charge carrier trapping,3,42 our comparison of the results from INS to IXS highlights the importance of careful chemical processing of the surface of nanomaterials.

Having validated the IXS technique on NC solids and highlighted it benefits relative to other methods for measuring vibrational structure, we extend our measurement to other nanocrystal samples. We perform measurements on three $\mathrm{PbS} / \mathrm{X} \mathrm{NC}$ thin films with different halide $(\mathrm{X}=$ $\mathrm{Cl}, \mathrm{Br}$, I) surface terminations, prepared from the same NC synthesis as the PbS/EDT thin film. We plot the extracted $\tilde{g}(\mathrm{E})$ from IXS (Figure 3a) along with the $\tilde{g}(\mathrm{E})$ computed from AIMD4 (Figure 3b).

From AIMD, we do not expect that the different terminations will change the number of the low frequency modes coming from the surface. Indeed, fitting a sum of two Gaussians to low energy ( $0 \mathrm{meV}<\hbar \omega<8 \mathrm{meV})$ portions of the $\tilde{g}(E)$ to all four surface terminations reveals 
values that are within error of one another (Supporting Information SI-3). However, AIMD calculations predict that the mean squared thermal displacement, $\left\langle u^{2}\right\rangle$, of the surface $\mathrm{Pb}$ atoms $\left\langle u_{P b}^{2}\right\rangle$, will decrease with increasing electronegativity of surface terminating atom due to stronger $\mathrm{Pb}-\mathrm{X}$ bonds: at $100 \mathrm{~K}$ the values predicted by AIMD are $\left\langle u_{P b}^{2}\right\rangle^{100 K}=0.029 \AA 2$ for EDT, $0.025 \AA 2$ for I, $0.024 \AA_{2}$ for $\mathrm{Br}$, and $0.023 \AA_{2}$ for $\mathrm{Cl}$, compared to $\left\langle u_{P b}^{2}\right\rangle_{B u l k}^{100 K}=0.018 \dot{\mathrm{A}}^{2} .4 \mathrm{As}$ described in Supporting Information SI-4, the $\left\langle u_{P b}^{2}\right\rangle$ for $\mathrm{Pb}$ atoms can be approximated from the IXS results to give $\left\langle u_{P b}^{2}\right\rangle^{300 K}=0.049 \pm 0.005 \AA 2$ for EDT, $0.043 \pm 0.002 \AA 2$ for I, $0.043 \pm$ $0.001 \AA_{2}$ for $\mathrm{Br}$, and $0.039 \pm 0.003 \AA_{2}$ for $\mathrm{Cl}$, in agreement with the trend predicted by the calculations.

Finally, we perform IXS measurements on thin films of cesium lead halide NCs,43-45 which of are of rapidly growing interest due to their performance in LEDs,46 solar cells47 and photodetectors. 48 The absorption spectrum and measured diffraction pattern of the $\mathrm{CsPbBr} 3$ NCs are given in Supporting Information SI-1. The extracted $\tilde{g}(\mathrm{E})$ is shown in Figure 4a. Within the error-bars, we can identify four peaks centered at $4.40 \pm 0.35 \mathrm{meV}, 8.00 \pm 0.35 \mathrm{meV}$, $11.60 \pm 0.35 \mathrm{meV}$, and $19.40 \pm 0.35 \mathrm{meV}$, the highest of which is expected to come from deformations of the halide octahedron about the lead atoms.49 For comparison, we perform AIMD simulations on bulk $\mathrm{CsPbBr} 3$, in the orthorhombic phase, which is expected at $300 \mathrm{~K}$ for bulk50 as well as as synthesized NCs51,52 (calculations for the cubic phase are provided in the Supporting Information SI-5). In Figure $4 \mathbf{b}$ and $\mathbf{c}$, we plot the computed $g(E)$, along with the partial $g_{X}(E)$ for each element, and the computed $\tilde{g}(E)$ using atomic factors $f_{C S}(q)=32.1, f_{P b}(q)=52.8, f_{B r}(q)=20.2$. Comparing the simulated and measured $\tilde{g}(E)$ from IXS, we see that the main features are common in both, noting that the peaks in the AIMD are shifted to lower energies due to the overestimation of the lattice constants in the calculations, 
$[a, b, c]_{A I M D}=[8.559,11.9,8.269] \AA$, compared to experiment $[a, b, c]=[8.255,11.759,8.207]$ $\AA .53$

In the $\tilde{g}(E)$ measured on $\mathrm{CsPbBr} 3 \mathrm{NCs}$, we note the presence of vibrations between $12 \mathrm{meV}$ and $19 \mathrm{meV}$, which are not found in the $\tilde{g}(E)$ calculated for bulk $\mathrm{CsPbBr}$. To find an explanation for these vibrations, we perform AIMD simulations on a $\sim(3.6 \mathrm{~nm}){ }_{3} \mathrm{CsPbBr} 3 \mathrm{NC}$. As shown in the Supporting Information SI-5, while the partial density of states of the center part of the $\mathrm{NC}$ resembles that of bulk $\mathrm{CsPbBr} 3$, the partial density of states of the outer three atomic layers of the NCs (dashed line in Figure 4c) shows a pronounced feature $\sim 14-15 \mathrm{meV}$. We therefore conclude that vibrations in our IXS measurement in that energy range come from localized vibrations on the surface of the NCs. Understanding how these surface modes can couple to charge carriers can for example yield insight into the atomistic origins of recombination at surfaces in perovskite solar cells and how to best passivate these modes.

The vibrational density of states, $g(E)$, of nanomaterials will, in general, differ from their bulk counterparts as a result of their large surface to volume ratio. Characterization of $g(E)$ constitutes the first step in moving towards a comprehensive understanding of phonon mediated transitions and thermodynamic properties in these materials. We have demonstrated that IXS can be used to efficiently probe the $g(E)$ of NCs in thin films. This technique should be readily applicable to other NC systems (e.g., metal NCs, core-shell NCs) and higherdimensional nanomaterials (e.g., nanowires, nanoplatelets).

\section{Methods}

\section{PbS Synthesis and Film Preparation}

Colloidal oleic-acid capped $r=1.6 \mathrm{~nm} \mathrm{PbS} \mathrm{NCs} \mathrm{(3.2} \mathrm{nm} \mathrm{in} \mathrm{diameter)} \mathrm{are} \mathrm{synthesized} \mathrm{using} \mathrm{the}$ hot injection method, where size-tunability is primarily achieved via different concentrations of oleic acid in the starting solution.54 The NCs are washed 3 times in mixtures of ethanol and methanol, and finally suspended in hexane at a concentration of $40 \mathrm{mg} / \mathrm{mL}$. Ligand exchange 
solutions consist of $3 \mathrm{mM}$ EDT and anhydrous acetonitrile, and $30 \mathrm{mM}$ of tetrabutylammonium iodide/ bromide/chloride in anhydrous methanol. Films are prepared in air via two identical and sequential spin casting steps. For both steps, $\sim 20 \mu \mathrm{L}$ of the NC solution was dropped on the substrate followed by a $1500 \mathrm{rpm}$ spin for $60 \mathrm{~s} . \sim 50 \mu \mathrm{L}$ of the ligand exchange solution is then dropped on the film, and after a $30 \mathrm{~s}$ wait, is spun off at $1500 \mathrm{rpm}$. Three rinsing steps are then repeated, flooding the film with the ligand exchange solvent and spinning dry at 1500 rpm.

\section{CsPbBrz Synthesis and Film Preparation}

Colloidal $\mathrm{CsPbBr} 3 \mathrm{NCs}(\sim 9.3 \mathrm{~nm}$ in diameter) are synthesized accordingly to previous publication.55 Briefly, freshly-made warm Cs-oleate solution is injected into the reaction mixture, containing $\mathrm{PbBr} 2$ and a mixture of coordinating solvents, at $180^{\circ} \mathrm{C}$. The reaction flask is rapidly cooled to room temperature in $5 \mathrm{~s}$ using ice-water bath. The NCs are purified by centrifuging of crude solution. NC precipitates are re-dissolved in hexane and precipitated with acetone, after what $\mathrm{NCs}$ are suspended in hexane at a concentration of $40 \mathrm{mg} / \mathrm{mL}$. $\mathrm{CsPbBr} 3$ NCs are covered with a mixture of oleic acid and oleylamine ligands. Films are prepared in air via spinning of $\mathrm{CsPbBr} 3 \mathrm{NC}$ solution, similarly to as described above. No ligand-exchange is performed for $\mathrm{CsPbBr}_{3} \mathrm{NCs}$. The as prepared films are then stores in inert nitrogen atmosphere.

\section{IXS Measurement}

Measurements are performed in vacuum in the grazing incident geometry, with the samples aligned with respect to the incoming x-rays such that the incident angle are just above the critical angle of the thin films, determined by maximizing the measured elastic scattering intensity of the strongest Bragg peak for each sample. Once alignment of the sample is complete, we measure the XRD spectrum of the thin film to determine a suitable range of $2 \theta$ to position the analyzers for the measurement of scattering, such that we measure at large $q$ (large $2 \theta$ ) and that the range contains no Bragg diffraction peak. For the PbS NCs, we find a 
suitable $2 \theta$ range between the [400] and [331] Bragg peaks of PbS centered at $2 \theta=34.4 \mathrm{o}$, corresponding to a $q \sim 53 \mathrm{~nm}-1>>\pi / a P b S \sim 5.3 \mathrm{~nm}-1$.

To calculate the density of state from the scattering signal, the elastic scattering intensity, $I_{E L}(E)$, must be rationally subtracted from the measured scattering intensity, $I_{M}(E)$, to obtain the inelastic scattering $I_{I N}(E) . \tilde{g}(E)$ can then be computed from $I_{I N}(E)$ upon deconvolution with the instrument response function, and normalization according to the thermal occupation factor of the vibrations. The ID28 beamline utilizes an array of 9 independent analyzers/detectors, spanning a $2 \theta=\sim 6$ o range. The analysis procedure outlined in SI-2 is followed for each of the 9 analyzers independently, and for the final $\tilde{g}(E)$ we average over all analyzers, keeping only the data on the energy gain side.

AIMD of $\mathrm{Cs} P b B r 3$

AIMD of PbS NCs and the phonon density of states for bulk PbS for reference are taken from Yazdani et al.4 while all calculations for $\mathrm{CsPbBr} 3$ are new to this work. Geometry and cell optimization, and ab initio molecular dynamics (AIMD) are performed within the $\mathrm{CP} 2 \mathrm{~K}$ program suite utilizing the quickstep module. 56 Calculations are carried out using a dual basis of localized Gaussians and plane-waves, 57 with a 300Ry plane-wave cutoff. As in previous calculations for $\mathrm{CsPbBr} 3 \mathrm{NCs,51}$ Double-Zeta-Valence-Polarization (DZVP),58 GoedeckerTeter-Hutter pseudopotentials59 for core electrons, and the Perdew-Burke-Ernzerhof (PBE) exchange correlation functional are used for all calculations. Convergence to 10-8 in SelfConsistent Field calculations is always enforced.

For $\mathrm{CsPbBr} 3$ bulk calculations, the orthorhombic and cubic unit cell lattice constants were determined through cell optimization, resulting in the lattice constants: $a=8.559 \AA, b=11.9 \AA$, $c=8.269 \AA$, and $a=b=c=6.019 \AA$ respectively. Geometry optimization is performed with the Quickstep module utilizing a Broyden-Fletcher-Goldfarb-Shannon (BFGS) optimizer. A 
maximum force of 24 meV $\AA-1$ is used as convergence criteria. All atoms in all systems are relaxed.

The $\mathrm{CsPbBr} 3 \mathrm{NC}$ was constructed according the atomistic model of ten Brinck et al.51 where the cubic $\mathrm{NC}$ with sides $=\sim 3.6 \mathrm{~nm}$ in length were cut from bulk $\mathrm{CsPbBr}$. Cs atoms were randomly removed from the surface of the $\mathrm{NC}$ to maintain charge compensation (i.e. $N_{C s}+$ $2 N P b-N B r=0)$, starting with the corners of the NC.

AIMD is performed in the canonical ensemble, using a CSVR thermostat, which achieves canonical sampling through velocity rescaling.60 For thermalization and calibration of the thermostat, the time constant of the thermostat is set to $15 \mathrm{fs}$ and the AIMD is run for $1 \mathrm{ps}$. The time constant is then set to $1 \mathrm{ps}$ for the remainder of the AIMD. All AIMD steps prior to equilibration of the total energy and temperature (typically $\sim 2 \mathrm{ps}$ ) are discarded. AIMD time steps of 10fs are used. The partial and total density of phonon states were obtained from the power spectrum of the mass-weighted position correlation function, $r_{i}(t): 61$

$$
g_{i}(E)=M_{i} E^{2}\left|\mathrm{~F}\left\{r_{i}(t)\right\}\right|^{2}, \quad g(E)=\sum_{i} g_{i}(E)
$$

where $M_{i}$ is the mass of atom $i$, and $r_{i}(t)$ are the time dependent coordinates extracted from AIMD.

Note, that the discrepancy between the position of the TA peak from AIMD with that calculated from bulk $\mathrm{PbS}$ and extracted from IXS and INS stems from the overestimation of the $\mathrm{Pb}-\mathrm{S}$ bond lengths in the AIMD calculations (6.0115 $)$, compared to its experimentally measured value (5.936 ), as further discussed in the Supporting Information (SI-3).

\section{ASSOCIATED CONTENT}

Supporting Information The Supporting Information is available free of charge via the Internet at http://pubs.acs.org. Absorption and x-ray diffraction spectra for all samples, 
detailed description of IXS analysis, phonon density of states of bulk $\mathrm{PbS}$ and $\mathrm{CsPbBr} 3, \mathrm{PbS}$ and $\mathrm{Cs} \mathrm{PbBr} 3 \mathrm{NCs}$, and mean square thermal displacements for PbS NCs are given.

\section{Corresponding Author}

Email:vwood@ethz.ch

\section{Funding Sources}

The authors acknowledge support from the Swiss National Science Foundation through the Quantum Sciences and Technology NCCR and an ETH Research Grant. Computations were supported by a grant from the Swiss National Supercomputing Centre (CSCS; project ID s674). The IXS experiments were performed on beamline ID28 at the European Synchrotron Radiation Facility (ESRF), Grenoble, France. The authors declare no competing financial interest.

\section{References}

(1) Nelson, T.; Fernandez-Alberti, S.; Roitberg, A. E.; Tretiak, S. Electronic Delocalization, Vibrational Dynamics, and Energy Transfer in Organic Chromophores. J. Phys. Chem. Lett. 2017, 8, 3020-3031.

(2) Huang, K.; Rhys, A. Theory of Light Absorption and Non-Radiative Transitions in FCentres. Proc. R. Soc. Lond. A. Math. Phys. Sci. 1950, 204, 406-423.

(3) Bozyigit, D.; Yazdani, N.; Yarema, M.; Yarema, O.; Lin, W. M. M.; Volk, S.;

Vuttivorakulchai, K.; Luisier, M.; Juranyi, F.; Wood, V. Soft Surfaces of Nanomaterials Enable Strong Phonon Interactions. Nature 2016, 531, 618-622.

(4) Yazdani, N.; Bozyigit, D.; Vuttivorakulchai, K.; Luisier, M.; Wood, V. Tuning Electron-Phonon Interactions in Nanocrystals through Surface Termination. Nano Lett. 2018, accepted, 1611.09930.

(5) Schaller, R. D.; Pietryga, J. M.; Goupalov, S. V.; Petruska, M. A.; Ivanov, S. A.; 
Klimov, V. I. Breaking the Phonon Bottleneck in Semiconductor Nanocrystals via Multiphonon Emission Induced by Intrinsic Nonadiabatic Interactions. Phys. Rev. Lett. $\mathbf{2 0 0 5}, 95,1-4$.

(6) Ridley, B. K. Multiphonon, Non-Radiative Transition Rate for Electrons in Semiconductors and Insulators. J. Phys. C Solid State Phys. 1978, 11, 2323.

(7) Benduhn, J.; Tvingstedt, K.; Piersimoni, F.; Ullbrich, S.; Fan, Y.; Tropiano, M.;

McGarry, K. A.; Zeika, O.; Riede, M. K.; Douglas, C. J.; et al. Intrinsic Non-Radiative Voltage Losses in Fullerene-Based Organic Solar Cells. Nat. Energy 2017, 2, 17053.

(8) Lin, S. H.; Chang, C. H.; Liang, K. K.; Chang, R.; Shiu, Y. J.; Zhang, J. M.; Yang, T.S.; Hayashi, M.; Hsu, F. C. Ultrafast Dynamics and Spectroscopy of Bacterial Photosynthetic Reaction Centers. In Advances in Chemical Physics; John Wiley \& Sons, Inc.: New York, USA; pp. 1-88.

(9) Bixon, M.; Jortner, J. Electron Transfer-from Isolated Molecules to Biomolecules. In Advances in Chemical Physics; 1999; pp. 35-202.

(10) Ulstrup, J.; Jortner, J. The Effect of Intramolecular Quantum Modes on Free Energy Relationships for Electron Transfer Reactions. J. Chem. Phys. 1975, 63, 4358-4368.

(11) Nan, G.; Yang, X.; Wang, L.; Shuai, Z.; Zhao, Y. Nuclear Tunneling Effects of Charge Transport in Rubrene, Tetracene, and Pentacene. Phys. Rev. B 2009, 79, 115203.

(12) Moser, C. C.; Keske, J. M.; Warncke, K.; Farid, R. S.; Dutton, P. L. Nature of Biological Electron Transfer. Nature 1992, 355, 796-802.

(13) Campos, M. P.; Hendricks, M. P.; Beecher, A. N.; Walravens, W.; Swain, R. A.; Cleveland, G. T.; Hens, Z.; Sfeir, M. Y.; Owen, J. S. A Library of Selenourea Precursors to PbSe Nanocrystals with Size Distributions near the Homogeneous Limit. J. Am. Chem. Soc. 2017, 139, 2296-2305.

(14) Caram, J. R.; Bertram, S. N.; Utzat, H.; Hess, W. R.; Carr, J. A.; Bischof, T. S.; 
Beyler, A. P.; Wilson, M. W. B.; Bawendi, M. G. PbS Nanocrystal Emission Is Governed by Multiple Emissive States. Nano Lett. 2016, 16, 6070-6077.

(15) Szilagyi, E.; Wittenberg, J. S.; Miller, T. A.; Lutker, K.; Quirin, F.; Lemke, H.; Zhu, D.; Chollet, M.; Robinson, J.; Wen, H.; et al. Visualization of Nanocrystal Breathing Modes at Extreme Strains. Nat. Commun. 2015, 6, 6577.

(16) Lindenberg, A. M.; Johnson, S. L.; Reis, D. A. Visualization of Atomic-Scale Motions in Materials via Femtosecond X-Ray Scattering Techniques. Annu. Rev. Mater. Res 2017, 47, 425-449.

(17) McKimmie, L. J.; Lincoln, C. N.; Jasieniak, J.; Smith, T. A. Three-Pulse Photon Echo Peak Shift Measurements of Capped CdSe Quantum Dots. J. Phys. Chem. C 2010, $114,82-88$.

(18) Lovesey, S. W. Theory of Neutron Scattering from Condensed Matter; Oxford University Press, 1984.

(19) Bosak, A.; Chernyshov, D.; Wehinger, B.; Winkler, B.; Le Tacon, M.; Krisch, M. Inbetween Bragg Reflections: Thermal Diffuse Scattering and Vibrational Spectroscopy with X-Rays. J. Phys. D. Appl. Phys. 2015, 48, 504003.

(20) Bosak, A.; Krisch, M. Phonon Density of States Probed by Inelastic X-Ray Scattering. Phys. Rev. B - Condens. Matter Mater. Phys. 2005, 72, 1-9.

(21) Bozyigit, D.; Yazdani, N.; Yarema, M.; Yarema, O.; Lin, W. M. M.; Volk, S.; Vuttivorakulchai, K.; Luisier, M.; Juranyi, F.; Wood, V. Soft Surfaces of Nanomaterials Enable Strong Phonon Interactions. Nature 2016, 531, 618-622.

(22) Balandin, A.; Wang, K. L.; Kouklin, N.; Bandyopadhyay, S. Raman Spectroscopy of Electrochemically Self-Assembled CdS Quantum Dots. Appl. Phys. Lett. 2000, 76, $137-139$.

(23) Gammon, D.; Brown, S. W.; Snow, E. S.; Kennedy, T. A.; Katzer, D. S.; Park, D. 
Nuclear Spectroscopy in Single Quantum Dots: Nanoscopic Raman Scattering and Nuclear Magnetic Resonance. Science (80-. ). 1997, 277, 85-88.

(24) Girard, A.; Saviot, L.; Pedetti, S.; Tessier, M. D.; Margueritat, J.; Gehan, H.; Mahler, B.; Dubertret, B.; Mermet, A. The Mass Load Effect on the Resonant Acoustic Frequencies of Colloidal Semiconductor Nanoplatelets. Nanoscale 2016, 8, 1325113256.

(25) Krauss, T. D.; Wise, F. W. Raman-Scattering Study of Exciton-Phonon Coupling in PbS Nanocrystals. Phys. Rev. B 1997, 55, 9860-9865.

(26) Milekhin, A.; Sveshnikova, L.; Duda, T.; Surovtsev, N.; Adichtchev, S.; Ding, L.; Zahn, D. R. T. Vibrational Spectra of Quantum Dots Formed by Langmuir-Blodgett Technique. J. Vac. Sci. Technol. B Nanotechnol. Microelectron. Mater. Process. Meas. Phenom. Appl. Phys. Lett. 2010, 28, 5-22.

(27) Mork, A. J.; Lee, E. M. Y.; Dahod, N. S.; Willard, A. P.; Tisdale, W. A. Modulation of Low-Frequency Acoustic Vibrations in Semiconductor Nanocrystals through Choice of Surface Ligand. J. Phys. Chem. Lett. 2016, acs.jpclett.6b01659.

(28) Miscuglio, M.; Lin, M.-L.; Di Stasio, F.; Tan, P.-H.; Krahne, R. Confined Acoustic Phonons in Colloidal Nanorod Heterostructures Investigated by Nonresonant Raman Spectroscopy and Finite Elements Simulations. Nano Lett. 2016, 16, 7664-7670.

(29) Yarema, M.; Yarema, O.; Lin, W. M. M.; Volk, S.; Yazdani, N.; Bozyigit, D.; Wood, V. Upscaling Colloidal Nanocrystal Hot-Injection Syntheses via Reactor Underpressure. Chem. Mater. 2016, 29, 796-803.

(30) Sears, V. F. Neutron Scattering Lengths and Cross Sections. Neutron News 1992, 3, $26-37$.

(31) Boles, M. A.; Ling, D.; Hyeon, T.; Talapin, D. V. The Surface Science of Nanocrystals. Nat. Mater. 2016, 15, 141-153. 
(32) Talapin, D. V; Lee, J.-S.; Kovalenko, M. V; Shevchenko, E. V. Prospects of Colloidal Nanocrystals for Electronic and Optoelectronic Applications. Chem. Rev. 2010, 110, $389-458$.

(33) Goodwin, E. D.; Diroll, B. T.; Oh, S. J.; Paik, T.; Murray, C. B.; Kagan, C. R. Effects of Post-Synthesis Processing on CdSe Nanocrystals and Their Solids: Correlation between Surface Chemistry and Optoelectronic Properties. J. Phys. Chem. C 2014, $118,27097-27105$.

(34) Hassinen, A.; Moreels, I.; De Nolf, K.; Smet, P. F.; Martins, J. C.; Hens, Z. ShortChain Alcohols Strip X-Type Ligands and Quench the Luminescence of PbSe and CdSe Quantum Dots, Acetonitrile Does Not. J. Am. Chem. Soc. 2012, 134, 2070520712.

(35) Kirmani, A. R.; Carey, G. H.; Abdelsamie, M.; Yan, B.; Cha, D.; Rollny, L. R.; Cui, X.; Sargent, E. H.; Amassian, A. Effect of Solvent Environment on ColloidalQuantum-Dot Solar-Cell Manufacturability and Performance. Adv. Mater. 2014, 26, $4717-4723$.

(36) Baron, A. Q. R. Introduction to High-Resolution Inelastic X-Ray Scattering. arXiv 2015, 1504.01098.

(37) Baron, A. Phonons in Crystals Using Inelastic X-Ray Scattering. arXiv Prepr.

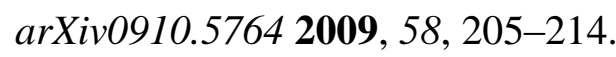

(38) Chuang, C.-H. M.; Brown, P. R.; Bulović, V.; Bawendi, M. G. Improved Performance and Stability in Quantum Dot Solar Cells through Band Alignment Engineering. Nat. Mater. 2014, 13, 796-801.

(39) Brown, P. R.; Kim, D.; Lunt, R. R.; Zhao, N.; Bawendi, M. G.; Grossman, J. C.; Bulovi??, V. Energy Level Modification in Lead Sulfide Quantum Dot Thin Films through Ligand Exchange. ACS Nano 2014, 8, 5863-5872. 
(40) Cromer, D. T.; Mann, J. B. X-Ray Scattering Factors Computed from Numerical Hartree-Fock Wave Functions. Acta Crystallogr. Sect. A 1968, 24, 321-324.

(41) Kohn, V. G.; Chumakov, A. I. DOS: Evaluation of Phonon Density of States from Nuclear Resonant Inelastic Absorption. Hyperfine Interact. 2000, 125, 205-221.

(42) Bozyigit, D.; Volk, S.; Yarema, O.; Wood, V. Quantification of Deep Traps in Nanocrystal Solids, Their Electronic Properties, and Their Influence on Device Behavior. Nano Lett. 2013, 13, 5284-5288.

(43) Protesescu, L.; Yakunin, S.; Bodnarchuk, M. I.; Krieg, F.; Caputo, R.; Hendon, C. H.; Yang, R. X.; Walsh, A.; Kovalenko, M. V. Nanocrystals of Cesium Lead Halide Perovskites ( $\mathrm{CsPbX} 3, \mathrm{X}=\mathrm{Cl}, \mathrm{Br}$, and I): Novel Optoelectronic Materials Showing Bright Emission with Wide Color Gamut. Nano Lett. 2015, 15, 3692-3696.

(44) Nedelcu, G.; Protesescu, L.; Yakunin, S.; Bodnarchuk, M. I.; Grotevent, M. J.; Kovalenko, M. V. Fast Anion-Exchange in Highly Luminescent Nanocrystals of Cesium Lead Halide Perovskites (CsPbX $3, \mathrm{X}=\mathrm{Cl}, \mathrm{Br}$, I).

(45) Weidman, M. C.; Seitz, M.; Stranks, S. D.; Tisdale, W. A. Highly Tunable Colloidal Perovskite Nanoplatelets through Variable Cation, Metal, and Halide Composition. ACS Nano 2016, 10, 7830-7839.

(46) Song, J.; Li, J.; Li, X.; Xu, L.; Dong, Y.; Zeng, H. Quantum Dot Light-Emitting Diodes Based on Inorganic Perovskite Cesium Lead Halides (CsPbX 3 ). Adv. Mater. 2015, 27, 7162-7167.

(47) Swarnkar, A.; Marshall, A. R.; Sanehira, E. M.; Chernomordik, B. D.; Moore, D. T.; Christians, J. A.; Chakrabarti, T.; Luther, J. M. Quantum Dot-Induced Phase Stabilization of -CsPbI3 Perovskite for High-Efficiency Photovoltaics. Science (80-. ). 2016, 354, 92-95.

(48) Yakunin, S.; Dirin, D. N.; Shynkarenko, Y.; Morad, V.; Cherniukh, I.; Nazarenko, O.; 
Kreil, D.; Nauser, T.; Kovalenko, M. V. Detection of Gamma Photons Using SolutionGrown Single Crystals of Hybrid Lead Halide Perovskites. Nat. Photonics 2016, 10, $585-589$.

(49) Huang, L.; Lambrecht, W. R. L. Lattice Dynamics in Perovskite Halides CsSnX3 with $\mathrm{X}=\mathrm{I}, \mathrm{Br}, \mathrm{Cl}$. Phys. Rev. B 2014, 90, 195201.

(50) Zhang, D.; Eaton, S. W.; Yu, Y.; Dou, L.; Yang, P. Solution-Phase Synthesis of Cesium Lead Halide Perovskite Nanowires. J. Am. Chem. Soc. 2015, 137, 9230-9233.

(51) ten Brinck, S.; Infante, I. Surface Termination, Morphology and Bright Photoluminescence of Cesium Lead Halide Perovskite Nanocrystals. ACS Energy Lett. 2016, acsenergylett.6b00595.

(52) Cottingham, P.; Brutchey, R. L. On the Crystal Structure of Colloidally Prepared CsPbBr 3 Quantum Dots. Chem. Commun. 2016, 52, 5246-5249.

(53) Rodová, M.; Brožek, J.; Knížek, K.; Nitsch, K. Phase Transitions in Ternary Cesium Lead Bromide. J. Therm. Anal. Calorim. 2003, 71, 667-673.

(54) Hines, M. a.; Scholes, G. D. Colloidal PbS Nanocrystals with Size-Tunable NearInfrared Emission: Observation of Post-Synthesis Self-Narrowing of the Particle Size Distribution. Adv. Mater. 2003, 15, 1844-1849.

(55) De Roo, J.; Ibáñez, M.; Geiregat, P.; Nedelcu, G.; Walravens, W.; Maes, J.; Martins, J. C.; Van Driessche, I.; Kovalenko, M. V.; Hens, Z. Highly Dynamic Ligand Binding and Light Absorption Coefficient of Cesium Lead Bromide Perovskite Nanocrystals. ACS Nano 2016, 10, 2071-2081.

(56) Vandevondele, J.; Krack, M.; Mohamed, F.; Parrinello, M.; Chassaing, T.; Hutter, J. Quickstep: Fast and Accurate Density Functional Calculations Using a Mixed Gaussian and Plane Waves Approach. Comput. Phys. Commun. 2005, 167, 103-128. (57) Lippert, G.; Hutter, J.; Parrinello, M. A Hybrid Gaussian and Plane Wave Density 
Functional Scheme. Mol. Phys. 1997, 92, 477-488.

(58) VandeVondele, J.; Hutter, J. Gaussian Basis Sets for Accurate Calculations on Molecular Systems in Gas and Condensed Phases. J. Chem. Phys. 2007, 127.

(59) Hartwigsen, C.; Goedecker, S.; Hutter, J. Relativistic Separable Dual-Space Gaussian Pseudopotentials from H to Rn. Phys. Rev. B 1998, 58, 3641-3662.

(60) Bussi, G.; Donadio, D.; Parrinello, M. Canonical Sampling through Velocity Rescaling. J. Chem. Phys. 2007, 126, 14101.

(61) Dove, M. T. Introduction to Lattice Dynamics; Cambridge University Press, 1993. 
a

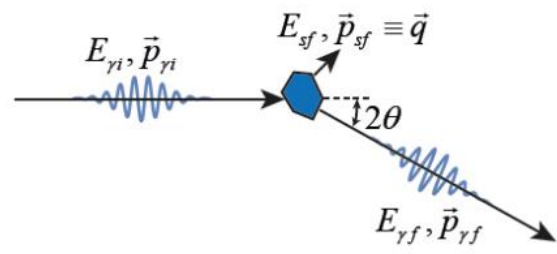

C

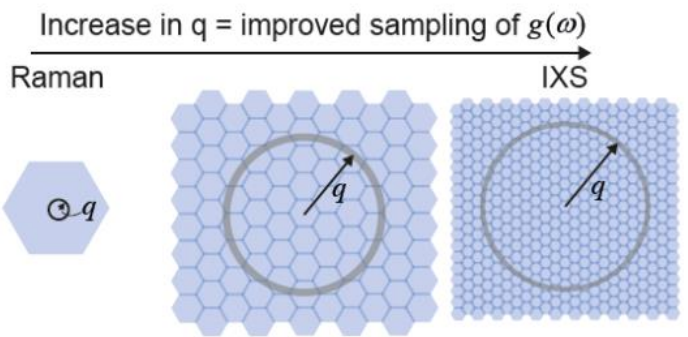

b
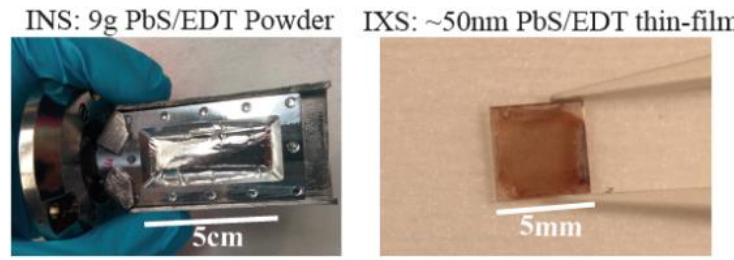

d

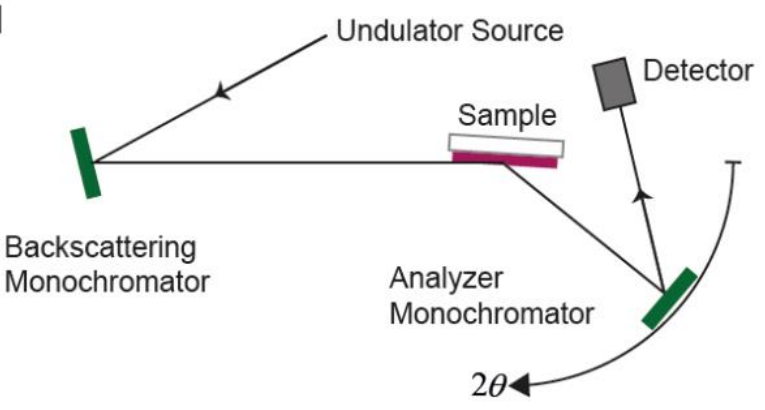

Figure 1: a) Schematic of energy and momentum conservation in an inelastic scattering process, where a probe of initial energy and momentum, $\left(E_{\gamma i}, \vec{p}_{\gamma i}\right)$, scatters from the sample to a final energy and momentum state, $\left(E_{\gamma f}, \vec{p}_{\gamma f}\right)$, transferring an energy, $E_{s f}=E_{\gamma i}-E_{\gamma f}$, and momentum, $\vec{p}_{s f} \equiv q=\vec{p}_{\gamma i}-\vec{p}_{\gamma f}$, to the sample. (b) Photographs of lead-sulfide nanocrystals (PbS NC) samples for both INS3 and IXS. Samples for INS were comprised of $\sim 9 \mathrm{~g}$ of NC-solid powder, the IXS samples consist of $\sim 50 \mathrm{~nm}$ of PbS NC-solid thin films on $5 \mathrm{~mm} \times 5 \mathrm{~mm}$ diamond substrates. c) Illustration of the dependence of the sampling of $g(E)$ of a polycrystalline material from IXS as a function of $q$, using a 2D hexagonal lattice as an example. d) Schematic of the setup of the IXS experiment at the ID28 beamline at ESRF. 

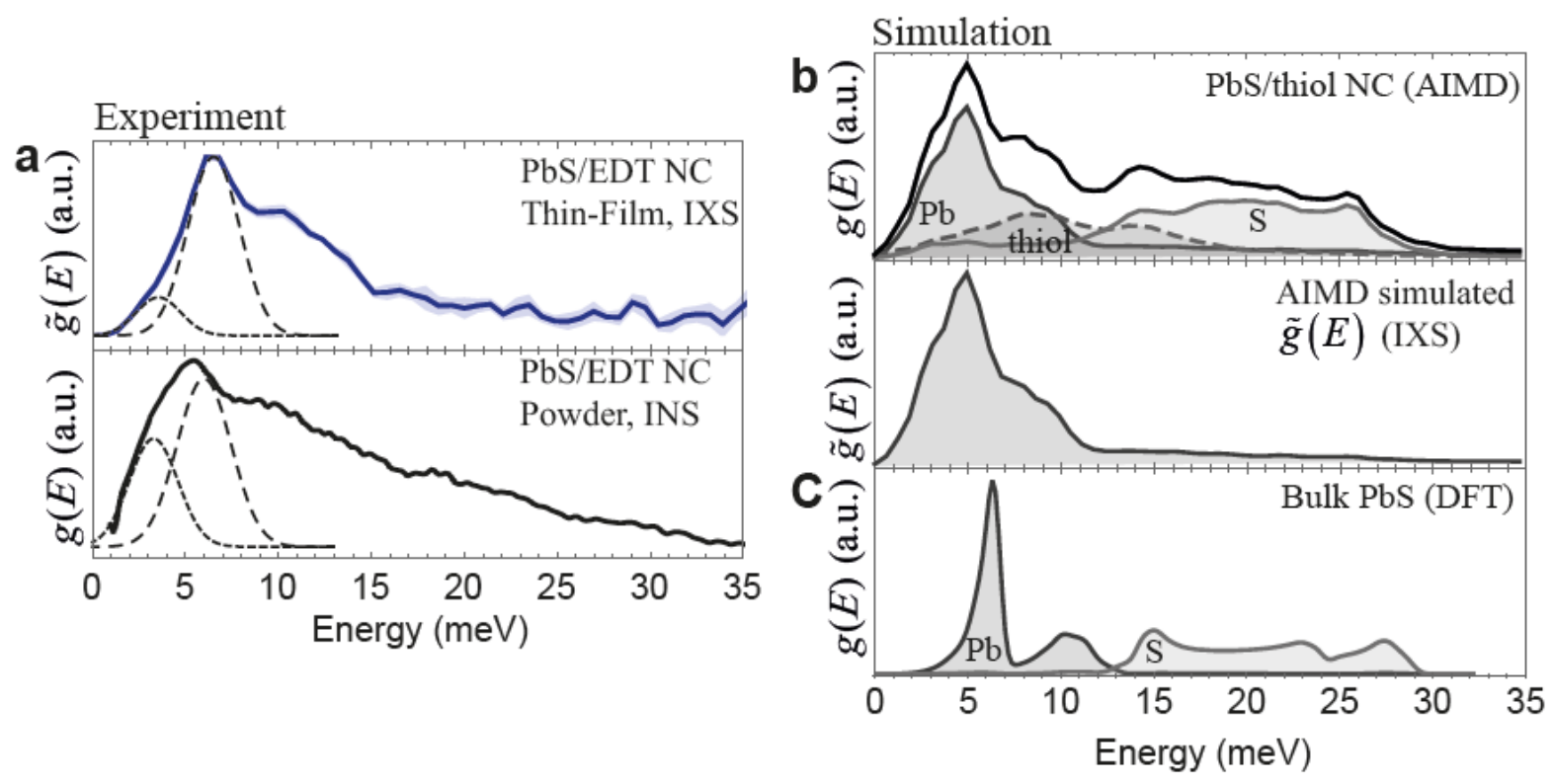

Figure 2: a) Top: the atomic structure factor weighted density of vibrational states, $\tilde{g}(E)$, extracted from IXS for the PbS/EDT NC-solid thin film. Error-bars represented by shading. Bottom: the density of vibrational states, $g(E)$, measured via INS on PbS/EDT NC-solid powder. The low energy portion of are fit with a sum of two Gaussians (black dashed lines). b) Top: AIMD calculated $g(E)$ for bulk $\mathrm{PbS}$ and a $1.2 \mathrm{~nm} \mathrm{PbS} /$ methanethiol together with partial $g(E)$ for each element. Bottom: The expected $\tilde{g}(E)$, computed from the AIMD results using eq. 1 , is also shown. c) DFT calculated $g(E)$ for bulk PbS. INS results and bulk PbS DFT calculations were adapted from Bozyigit et. al.3, while the AIMD results were adapted from Yazdani et. al.4 

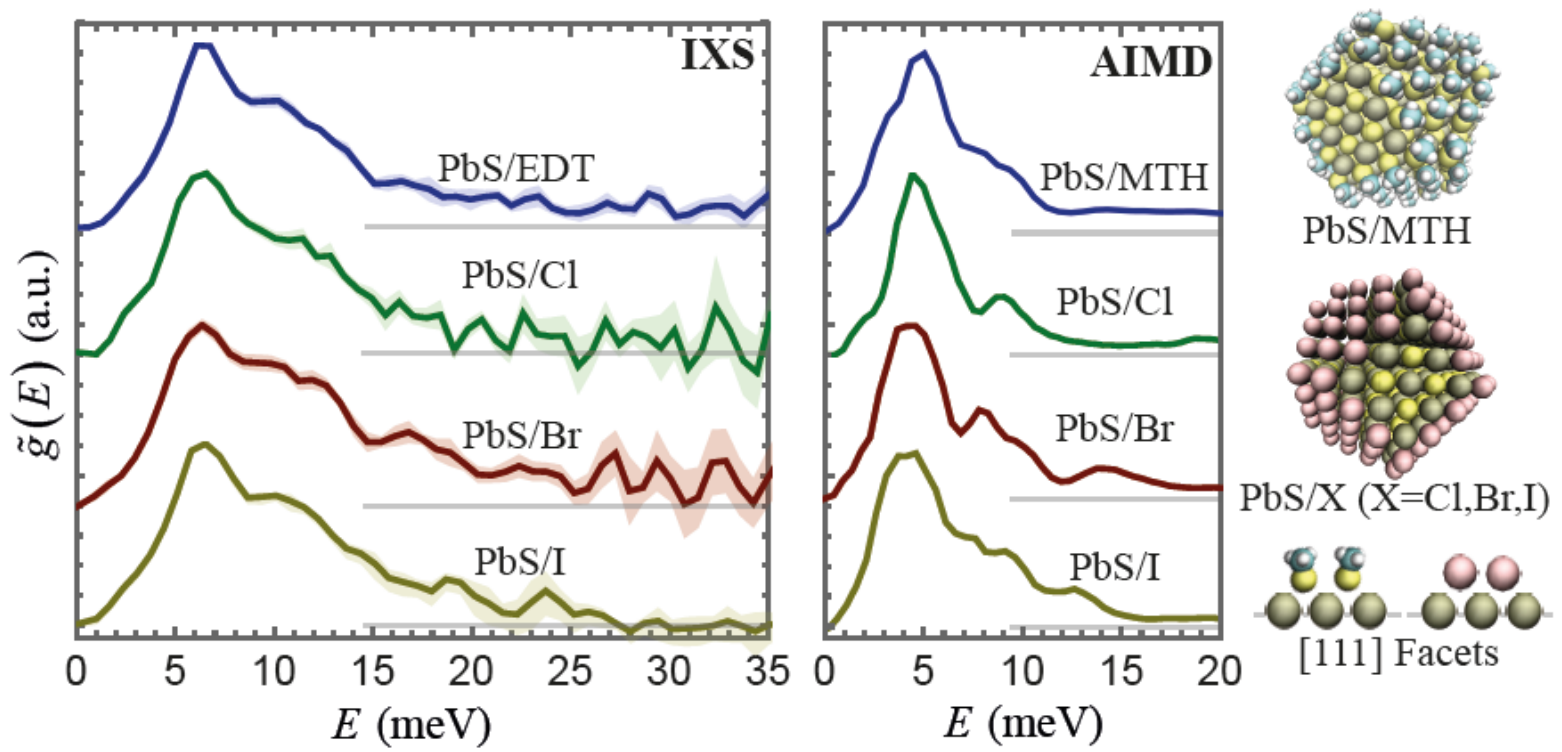

Figure 3: a) The atomic structure factor weighted density of vibrational states, $\tilde{g}(E)$, measured for IXS for the PbS NC-solid thin films with EDT, Cl, Br, and I surface termination (curves offset for clarity). Error-bars represented by shading. b) $\tilde{g}(E)$ expected for each computed from the AIMD results of $1.2 \mathrm{~nm}$ NCs4. The atomistic models for the AIMD calculations and a depiction of the surface termination on the [111] facets of the NCs is shown on the right. 


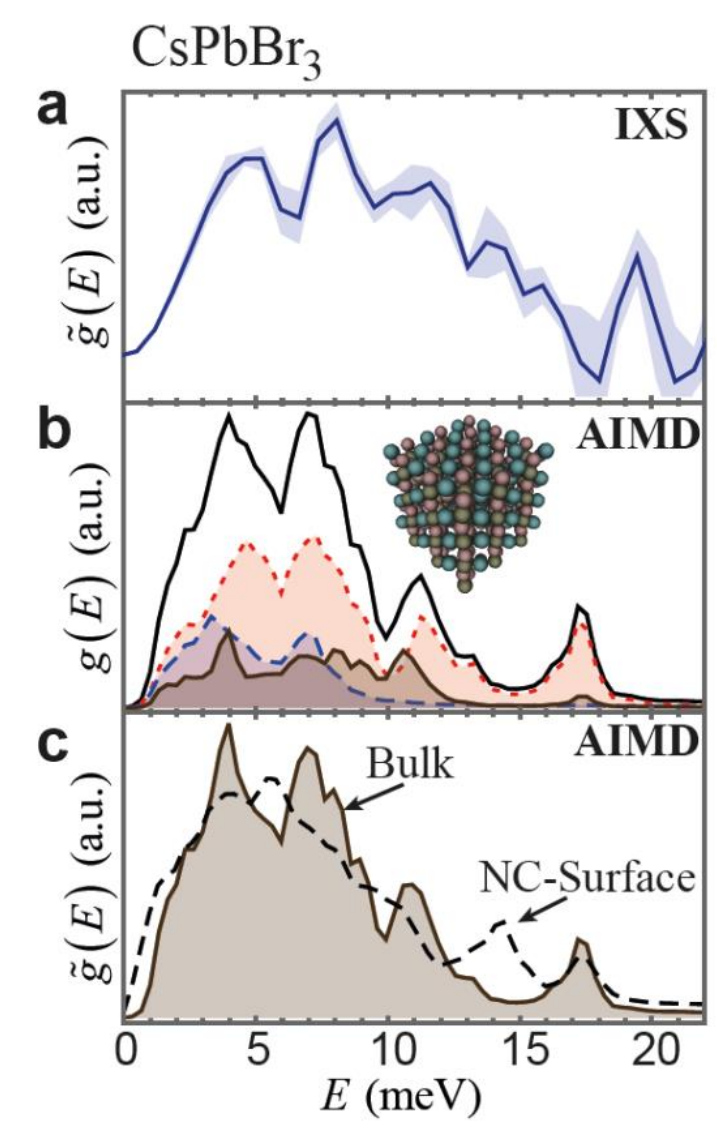

Figure 4: c) Vibrational density of states of $\mathrm{CsPbBr}_{3} \mathrm{NCs}, \tilde{g}(E)$, measured from IXS. Errors bars are represented by the shading. b) The phonon density of states for bulk $\mathrm{CsPbBr} 3$ (black line), in the orthorhombic phase, computed by AIMD along with the partial density of states for each atom type $(\mathrm{Cs}=$ blue dashed; $\mathrm{Pb}=$ brown; $\mathrm{Br}=$ red dotted $)$. The inset shows the $\mathrm{CsPbBr} 3$ crystal structure. c) The atomic structure factor weighted density of vibrational states, $\tilde{g}(E)$, for bulk $\mathrm{CsPbBr}_{3}$ (brown solid line) computed from the AIMD results to reflect what we expect from IXS measurements. The dashed line shows the atomic structure factor weighted partial density of vibrational states for the outer 3 atomic layers of a $\mathrm{CsPbBr} 3 \mathrm{NC}$ (see Supporting Information SI-5) 